\title{
Pre-clinical imaging of transgenic mouse models of neuroblastoma using a dedicated 3-element solenoid coil on a clinical 3T platform
}

\author{
Gilberto S Almeida ${ }^{1,3}$, Rafal Panek ${ }^{1,3}$, Albert Hallsworth ${ }^{2}$, Hannah Webber ${ }^{2}$, Efthymia Papaevangelou ${ }^{1}$, \\ Jessica KR Boult ${ }^{1}$, Yann Jamin ${ }^{1}$, Louis Chesler ${ }^{2}$ and Simon P Robinson ${ }^{*}, 1$ \\ ${ }^{1}$ Division of Radiotherapy \& Imaging, The Institute of Cancer Research, 15 Cotswold Road, Sutton, Surrey SM2 5NG, UK and \\ ${ }^{2}$ Division of Clinical Studies, The Institute of Cancer Research, 15 Cotswold Road, Sutton, Surrey, SM2 5NG, UK
}

Background: The use of clinical MRI scanners to conduct pre-clinical research facilitates comparisons with clinical studies. Here the utility and sensitivity of anatomical and functional MRI data/biomarkers acquired from transgenic mouse models of neuroblastoma using a dedicated radiofrequency (RF) coil on a clinical 3T scanner was evaluated.

Methods: Multiparametric MRI of transgenic mice bearing abdominal neuroblastomas was performed at $3 T$, and data cross-
referenced to that acquired from the same mice on a pre-clinical $7 \mathrm{~T} M R I$ system. $T_{2}$-weighted imaging, quantitation of the native
longitudinal relaxation time $\left(T_{1}\right)$ and the transverse relaxation rate $\left(R_{2}{ }^{\star}\right)$, and dynamic contrast-enhanced (DCE)-MRI, was used to
assess tumour volume, phenotype and response to cyclophosphamide or cabozantinib.

Results: Excellent $T_{2}$-weighted image contrast enabled clear tumour delineation at 3T. Significant correlations of tumour volume $(R=0.98, P<0.0001)$ and $R_{2}{ }^{*}(R=0.87, P<0.002)$ measured at 3 and $7 \mathrm{~T}$ were established. Mice with neuroblastomas harbouring the anaplastic lymphoma kinase mutation exhibited a significantly slower $R_{2}{ }^{*}(P<0.001)$, consistent with impaired tumour perfusion. DCE-MRI was performed simultaneously on three transgenic mice, yielding estimates of $K^{\text {trans }}$ for each tumour (median $K^{\text {trans }}$ values of $0.202,0.168$ and $\left.0.114 \mathrm{~min}^{-1}\right)$. Cyclophosphamide elicited a significant reduction in both tumour burden $(P<0.002)$ and native $T_{1}(P<0.01)$, whereas cabozantinib induced significant $(P<0.01)$ tumour growth delay.

Conclusions: Simultaneous multiparametric MRI of multiple tumour-bearing animals using this coil arrangement at 3T can provide high efficiency/throughput for both phenotypic characterisation and evaluation of novel therapeutics, and facilitate the introduction of functional MRI biomarkers into aligned imaging-embedded clinical trials.

High field $(>4.7 \mathrm{~T})$ small animal dedicated MRI systems that provide high resolution and signal to noise ratios (SNR) have and continue to be the preferred platform for pre-clinical imaging investigations. The physical contrast characteristics and imaging performance of such pre-clinical scanners differs substantially from clinical MRI systems. The use of clinical scanners to conduct pre-clinical research is attractive, as it facilitates a more direct comparison with clinical studies, the application and development of clinically applicable imaging protocols, matching of field strength-related mechanisms such as relaxation, and provides evidence to support the clinical relevance of functional MRI data/ biomarkers (Brockmann et al, 2007; Chen et al, 2007; Inderbitzin 
et al, 2007; Linn et al, 2007; Bilgen, 2013). This approach also benefits from the hardware and software advances continuously being made on modern clinical MRI systems, such as parallel transmit and receive technology, motion and contrast tracking, $\mathrm{k}$-space under-sampling and view-sharing strategies (Ullman et al, 2004; Wech et al, 2012; Jerome et al, 2016). Additionally, the generous homogeneous field region of clinical scanners allows for high quality, whole-body pre-clinical imaging, and the potential for simultaneous data acquisition from several subjects during the same scanning session (Dazai et al, 2004; Nieman et al, 2007). Nuclear relaxation times are also often better differentiated at relatively lower field strengths (de Graaf et al, 2006), leading to superior contrast between imaged soft tissues, crucial for reliable organ and pathology delineation.

Most clinical scanners operate at between 1 and 3T, having lower SNR levels than pre-clinical high field systems, and hence reduced image quality if standard clinical imaging coils are used. This is exacerbated particularly when imaging the small fields-ofview necessary while using rodents, but with a high enough resolution to be able to accurately resolve lesions and acquire meaningful functional data. One approach of increasing SNR is to use small dedicated radiofrequency (RF) receiver coils. Such coils can be designed to fit closely to the object of interest, giving a better coupling between the object and coil (high filling factor), a stronger signal and thus improved image quality.

In pre-clinical cancer research performed in vivo, transgenic mouse models, in which tumours are driven by expression of the target gene of interest and arise spontaneously within the native tissue of origin, are being increasingly exploited (de Jong et al, 2014). These models more faithfully emulate human tumour growth, tumour-host stromal interactions and vasculature, metastatic potential and therapeutic response in vivo. Such models demand non-invasive methods to longitudinally and accurately assess the progression and treatment response of tumours that typically arise within deep-seated anatomical locations.

The purpose of this study was to evaluate the utility and sensitivity of a non-bespoke high resolution RF coil for use on a clinical 3T scanner for the acquisition of anatomical and functional MRI data/biomarkers from transgenic mouse models of neuroblastoma in vivo.

\section{MATERIALS AND METHODS}

All procedures involving animals were performed in accordance with the local ethical review panel, the UK Home Office Animals (Scientific Procedures) Act 1986, the United Kingdom National Cancer Research Institute guidelines for the welfare of animals in cancer research and the ARRIVE guidelines (Workman et al, 2010; Kilkenny et al, 2010).

$T_{1}$ phantom. To assess the linearity of $T_{1}$ measurements on both the 3 and $7 \mathrm{~T}$ platforms, phantoms with increasing concentrations of gadolinium (from 30 to $500 \mathrm{~nm}$ ) were imaged in both scanners at ambient temperature. These solutions were transferred into $5 \mathrm{~mm}$ NMR tubes, which in turn were placed in $50 \mathrm{ml}$ falcon tubes and restrained using dental paste to limit motion artefacts. The phantoms were scanned four times on each scanner, and the average value used to assess the $T_{1}$ field dependence.

Transgenic mouse models and drug treatment. The aberrant expression of the transcription factor $M Y C N$ is a potent oncogenic stimulus in cancer. MYCN expression correlates with an aggressive tumour phenotype, enhanced tumour angiogenesis and poor clinical prognosis in neuroblastoma (Maris, 2010). Many paediatric cancers arise through the aberrant expression of just a few driver genes, and are thus amenable to transgenic mouse modelling approaches (Chesler and Weiss, 2011). For example, targeted overexpression of $M Y C N$ to the neural crest under the control of the tyrosine hydroxylase promoter resulted in the Th-MYCN transgenic mouse model in which abdominal tumours spontaneously develop that faithfully replicate the disease biology of highrisk neuroblastoma (Weiss et al, 1997). More recently, the identification of mutations in the anaplastic lymphoma kinase $(A L K)$ gene in neuroblastoma, and its close association with $M Y C N$ amplification, stimulated the development of a novel double transgenic mouse model for $A L K$ and MYCN (Berry et al, 2012). In both the Th-MYCN and Th- $A L K^{F 1174 L} / \mathrm{Th}-M Y C N$ transgenic mice, solid tumours spontaneously arise within the retroperitoneum in peri-renal and para-spinal abdominal regions, consistent with the typical clinical distribution and radiological presentation of human neuroblastoma.

In this study, male and female tumour-bearing Th-MYCN and Th- $A L K^{F 1174 L} /$ Th-MYCN mice, initially identified by palpation, underwent MRI at around 50 and 30 days old, respectively (Berry et al, 2012; Rasmuson et al, 2012). Their genotype was confirmed by analysing DNA from the tail using real-time quantitative reverse transcription polymerase chain reaction.

\section{MRI data acquisition}

Study protocol. Twenty-three transgenic mice (Th-MYCN $n=14$, Th-ALK ${ }^{F 1174 L} /$ Th-MYCN $n=9$ ) underwent imaging on the 3T platform. Of these, 10 mice (Th-MYCN $n=7$, Th- $A L K^{F 1174 L} / \mathrm{Th}$ MYCN $n=3$ ) were scanned at both 3 and $7 \mathrm{~T}$ within $24 \mathrm{~h}$ of each other in order to compare tumour volume determination and quantification of tumour $R_{2}^{*}$ at 3 and 7T. Dynamic contrastenhanced (DCE)-MRI was performed simultaneously on three Th$M Y C N$ mice at $3 \mathrm{~T}$. To evaluate the $3 \mathrm{~T}$ platform for imagingembedded intervention trials, anatomical and functional (native $T_{1}$ and $R_{2}{ }^{*}$ ) MRI measurements were acquired from Th-MYCN tumours prior to and $24 \mathrm{~h}$ post-treatment with either saline (control, $n=4$ ) or a single $25 \mathrm{mg} \mathrm{kg}^{-1}$ i.p. dose of the DNA alkylating agent cyclophosphamide (CPM, $n=5)$, or prior to and $48 \mathrm{~h}$ post treatment with a daily oral dose of either water (control, $n=4)$ or $30 \mathrm{mg} \mathrm{kg}^{-1}$ of the multi-kinase inhibitor cabozantinib (CBZ, $n=5)$ (Yakes et al, 2011).

$3 T$ data acquisition and analysis. MRI data was acquired on a clinical scanner (Philips Achieva, Best, Netherlands) using a dedicated high-resolution RF coil ('Mouse Hotel', Philips, Best, Netherlands) (Figure 1). The coil consists of three $40 \mathrm{~mm}$ inner diameter solenoids, with the long axis oriented perpendicular to the magnet $B_{0}$ field. This coil arrangement enables the simultaneous acquisition of MRI data from up to three animals using independent receiver channels. The $3 \mathrm{~T}$ imaging protocol, which included $T_{2}$-weighted, $T_{1}$ and $R_{2}{ }^{*}$ measurements, was tested and optimised using phantoms prior to the in vivo study. A compromise between SNR, spatial and temporal resolution was prioritised over a direct parameter match to pre-clinical $7 \mathrm{~T}$ protocols.

Anaesthesia was induced by intraperitoneal injection of midazolam $\left(5 \mathrm{mg} \mathrm{kg}^{-1}\right)$, fentanyl $\left(0.05 \mathrm{mg} \mathrm{kg}^{-1}\right)$, and medetomidine $\left(0.5 \mathrm{mg} \mathrm{kg}^{-1}\right)$ in sterile water. For $3 \mathrm{~T}$ data acquisition, up to three anaesthetised mice were positioned supine with their abdomens in the centre of each individual element of the coil. Mouse core body temperature was maintained by a built-in heating system controlled within the 'Mouse Hotel' coil.

Initially, whole body anatomical $T_{2}$-weighted images were acquired at $3 \mathrm{~T}$ using a coronal orientation with full anatomical coverage of all three animals (TSE, 20 slices, slice thickness: $1 \mathrm{~mm}$, $\mathrm{FOV}=250 \times 200 \mathrm{~mm}$, resolution $=0.2 \times 0.2 \mathrm{~mm}, \mathrm{ETL}=14, \mathrm{TE} /$ $\mathrm{TR}=80 / 3000 \mathrm{~ms}, \mathrm{NSA}=1, \mathrm{TA}=3 \mathrm{~min} 48 \mathrm{~s}$ ). $T_{2}$-weighted images were used to assess the extent of the disease and aid axial sequence planning, which was performed across the central part of the tumour. Proton density and $T_{1}$-weighted images were then 


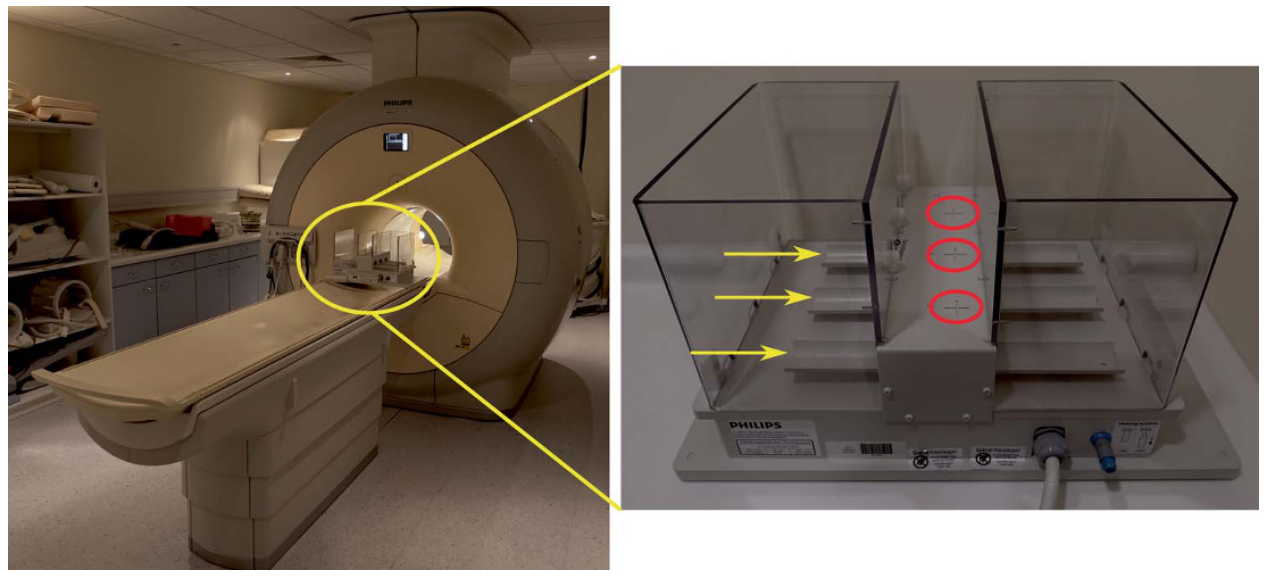

Figure 1. 'Mouse hotel' coil and the 3T clinical scanner. Photographs of the coil arrangement on the 3T scanner, and the high resolution 3channel/3-animal MR RF coil. The yellow arrows indicate the three individual mouse beds and the red circles the centre of each element of the coil. Positioning of the coil in the scanner as shown means the mice are perpendicular to the $B_{0}$ field.

acquired using a 3D spoiled gradient echo sequence with variable flip angle (axial orientation, 7 slices, slice thickness: $1.5 \mathrm{~mm}$, resolution $=0.25 \times 0.25 \mathrm{~mm}, \quad F O V=200 \times 85 \mathrm{~mm}, F A=3$ and $16^{\circ}, 10$ dummy scans, $\mathrm{TE} / \mathrm{TR}=2.3 / 7 \mathrm{~ms}, \mathrm{NSA}=1, \mathrm{TA}=27 \mathrm{~s}$ ). Multigradient echo (MGE) images were acquired using a $2 \mathrm{D}$ gradient echo sequence with multiple echo times (axial orientation, 3 slices, slice thickness: $1.5 \mathrm{~mm}$, resolution $=0.3 \times 0.3 \mathrm{~mm}$, $\mathrm{FOV}=200 \times 50 \mathrm{~mm}, \mathrm{FA}=24^{\circ}, \mathrm{TE}=4.6,11.5,18.4,25.3,32.2$ and $39.1 \mathrm{~ms}, \mathrm{TR}=500 \mathrm{~ms}, \mathrm{NSA}=1, \mathrm{TA}=30 \mathrm{~s}$ ). No fat suppression was employed.

For the acquisition of DCE-MRI data, a lateral tail vein of each mouse was cannulated with a $27 \mathrm{G}$ butterfly catheter connected to a $10 \mathrm{~m}$ long line of polyethylene tubing (BPTE-T10, $0.23^{\prime \prime}$ i.d., 0.38 " o.d.), and attached through the available wave-guides to syringe pumps located in the scanner control room, thereby enabling the simultaneous remote intravenous administration of contrast agent to each mouse. DCE-MRI data were acquired using the 3D spoiled gradient echo sequence with 200 time points, a temporal resolution of $2.5 \mathrm{~s}$ and a total acquisition time of 8 mins $33 \mathrm{~s}$. A bolus injection of $0.1 \mathrm{mmol} \mathrm{kg}^{-1}$ gadopentate dimeglumine (Gd-DTPA) was simultaneously administered to all three mice at a rate of $2 \mathrm{ml} \mathrm{min}^{-1}, 30 \mathrm{~s}$ after the start of the dynamic acquisition. The total $3 \mathrm{~T}$ protocol scanning time was less than 15 min.

Tumour volumes were extrapolated from manually drawn regions of interest (ROIs) on $T_{2}$-weighted images for each tumour-containing slice, where clear delineation of tumour could be determined, using OsiriX Lite (Pixmeo). The $T_{1}$-weighted images were analysed using dedicated software (MRIW, working under IDL, (d'Arcy et al, 2006)), and the ratio between the proton density $\left(\mathrm{FA}=3^{\circ}\right)$ and $T_{1}$-weighted $\left(\mathrm{FA}=16^{\circ}\right) \quad 3 \mathrm{D}$ spoiled gradient echo used to provide estimates of the native tumour spin-lattice relaxation time $T_{1}$ (ms) (Fram et al, 1987). The MGE data were analysed using another dedicated software (ADEPT, working under IDL (Doran et al, 2012)), with tumour $R_{2}^{*}\left(\mathrm{~s}^{-1}\right)$ maps calculated by fitting a single exponential to the signal intensity echo time curve on a voxel-by-voxel basis using a Bayesian maximum a posteriori approach (Walker-Samuel et al, 2010). MRIW was used to fit the DCE-MRI data incorporating the Kety model (Tofts et al, 1999) and a literature-derived murine arterial input function (Benjaminsen et al, 2004), providing estimates of $K^{\text {trans }}\left(\mathrm{min}^{-1}\right)$, the volume transfer constant between blood plasma and extracellular extravascular space, $V_{\mathrm{e}}$ (dimensionless volume fraction, and IAUGC $_{60}$ (mM.s), the initial area under the gadolinium concentration curve from 0 to $60 \mathrm{~s}$ after injection of Gd-DTPA.
Table 1. Longitudinal relaxation times quantified from the gadolinium phantoms at 3 and $7 T$

\begin{tabular}{|c|c|c|}
\hline [Gd] & 7T & 3T \\
\hline Saline & $2358 \pm 21$ & $1608 \pm 223$ \\
\hline 30 nм & $1811 \pm 34$ & $1238 \pm 84$ \\
\hline $40 \mathrm{~nm}$ & $1605 \pm 14$ & $1002 \pm 83$ \\
\hline $50 \mathrm{~nm}$ & $1499 \pm 15$ & $950 \pm 132$ \\
\hline $100 \mathrm{~nm}$ & $1227 \pm 178$ & $704 \pm 7$ \\
\hline $500 \mathrm{~nm}$ & $400 \pm 4$ & $241 \pm 3$ \\
\hline
\end{tabular}

$7 T$ data acquisition and analysis. Volumetric analysis and quantification of $T_{1}$ and $R_{2}{ }^{*}$ of tumours arising within Th-MYCN and Th- $A L K^{F 1174 L} /$ Th-MYCN transgenic mice was performed on a 7T horizontal bore microimaging system (Bruker Instruments, Ettlingen, Germany) using a $3-\mathrm{cm}$ birdcage volume coil, as previously described (Jamin et al, 2013, 2014).

Statistical analysis. Statistical analysis was performed using GraphPad Prism 6. The absolute values for tumour volume, and median values for $T_{1}$ and $R_{2}{ }^{*}$ were used. Any significant difference within the same group was identified using Student's two-tailed paired $t$-test, with a $5 \%$ level of significance. Any significant difference between groups after treatment was tested using an unpaired $t$-test. The linear correlation coefficient $R$ was used to test correlation strength, direction and linear association between volumes and relaxation times measured at both field strengths.

\section{RESULTS}

Measurements from the $T_{1}$ phantom for all Gd-DTPA concentrations at both field strengths are shown in Table 1. A highly significant correlation $(R=0.99, \quad P<0.0001)$ was found between the $T_{1}$ values obtained from the $3 \mathrm{~T}$ clinical scanner and the $7 \mathrm{~T}$ pre-clinical scanner, with the median $T_{1}$ values being higher at $7 \mathrm{~T}$.

Representative anatomical $T_{2}$-weighted $M R$ images of tumourbearing mice acquired at 3 and $7 \mathrm{~T}$ are displayed in Figure $2 \mathrm{~A}$ and $B$, revealing large peri-renal masses within each mouse abdomen, and occasionally an additional thoracic tumour, consistent with the radiological presentation of neuroblastoma. The excellent image 


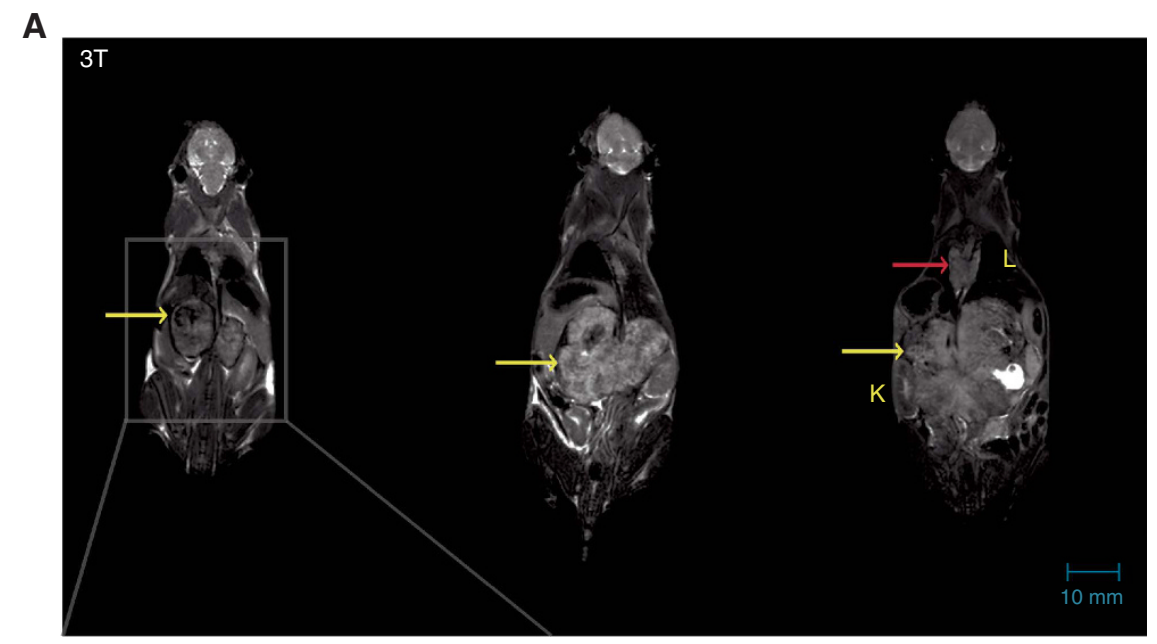

B
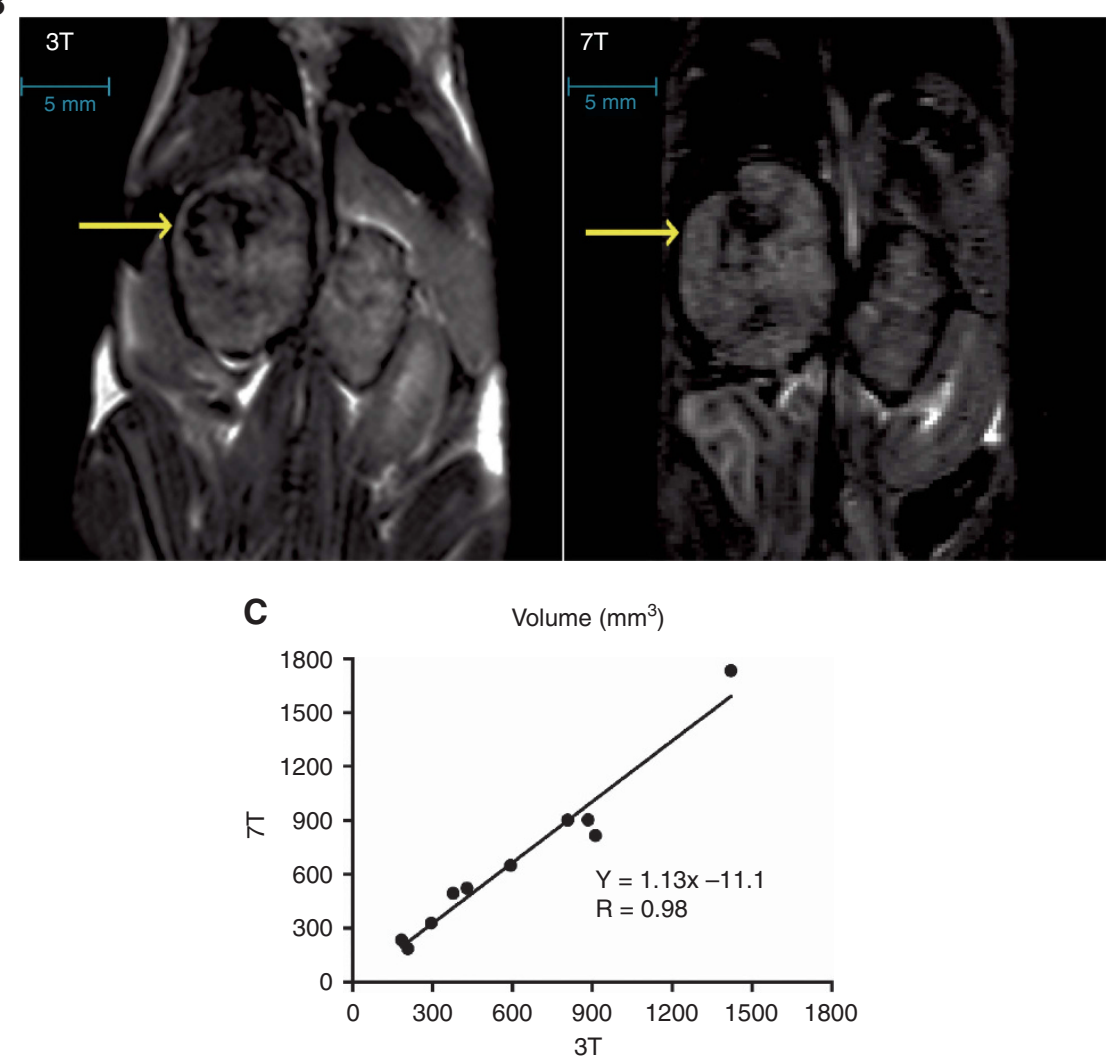

Figure 2. Anatomical imaging at 3 and 7T. (A) Anatomical $T_{2}$-weighted coronal images acquired simultaneously from three Th-MYCN mice with abdominal neuroblastoma using the high resolution 3-channel/3-animal MR RF coil on the 3T clinical scanner, with a scale bar in blue. Each tumour is indicated by yellow arrows, with kidney $(K)$ and lungs $(L)$ also annotated. An additional thoracic lesion identified in one of the mice is indicated with a red arrow. (B) Expanded $T_{2}$-weighted image of the abdomen of the first mouse in (A) acquired at $3 T$, and the corresponding $T_{2}$-weighted image acquired from the same mouse at 7T, with corresponding scale bars in blue. (C) Linear regression analysis of the volumetric measurements determined from $T_{2}$-weighted MRI of tumour-bearing transgenic mice imaged at 3 and $7 \mathrm{~T}$ revealed a positive and highly significant correlation $(R=0.98, P<0.0001)$.

contrast yielded clear tumour delineation across contiguous slices that could be used for accurate volumetric analysis in vivo. No significant difference was found between volumetric measurements acquired at the different field strengths (mean tumour volume $819 \pm 153 \mathrm{~mm}^{3}$ at $3 \mathrm{~T} v s 889 \pm 191 \mathrm{~mm}^{3}$ at $\left.7 \mathrm{~T}, n=10, P>0.05\right)$, and which were significantly correlated $(R=0.98, P<0.0001$, Figure 2C).

Parametric $R_{2}{ }^{*}$ maps from a tumour arising in a Th-MYCN mouse acquired at both 3 and $7 \mathrm{~T}$ are shown in Figure $3 \mathrm{~A}$. While anatomical $T_{2}$-weighted images revealed some anticipated differences in animal positioning, it was possible to identify similar tumour positions at both field strengths. A heterogeneous distribution of $R_{2}{ }^{*}$ values was apparent across all tumours imaged at both field strengths. A statistically significant positive correlation of $R_{2}^{*}$ values quantified at 3 and $7 \mathrm{~T}$ was determined $(n=10$, $R=0.87, P=0.0012$, Figure $3 \mathrm{~B}$ ). Unlike the phantom data, no significant correlation in tumour $T_{1}$ relaxation times measured at 3 and $7 \mathrm{~T}$ in vivo was found. This may be a consequence of different 
A

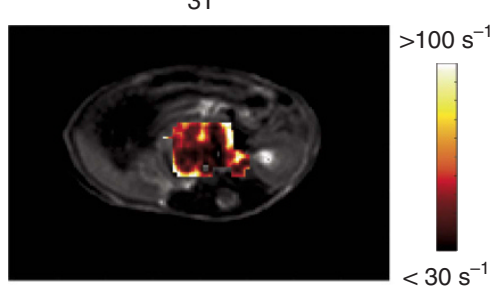

7T

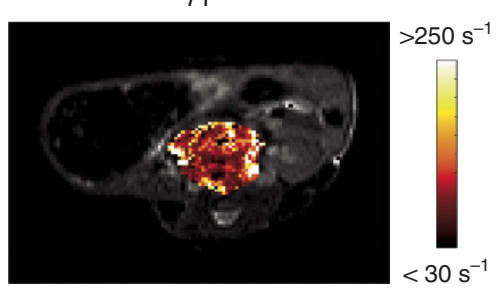

B

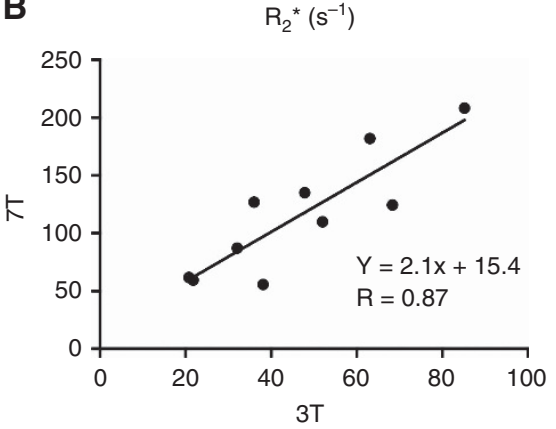

C

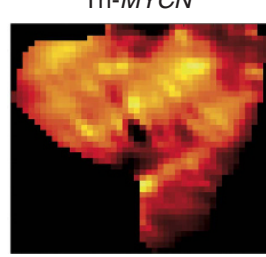

Th- $A L K^{F 1174 L, ~}$ Th-MYCN

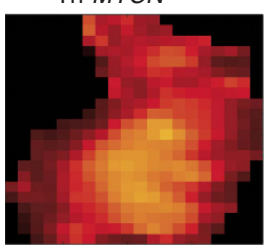

D

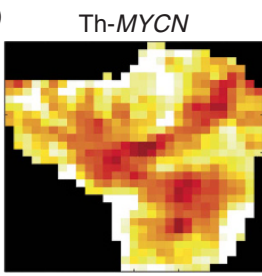

Th- $A L K^{F 1174 L}$, Th-MYCN

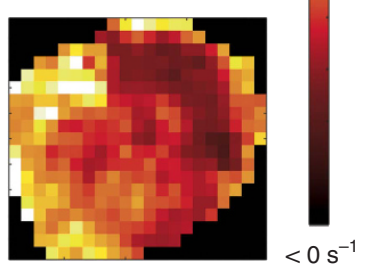

$>1500 \mathrm{~ms}$

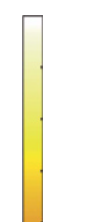

E

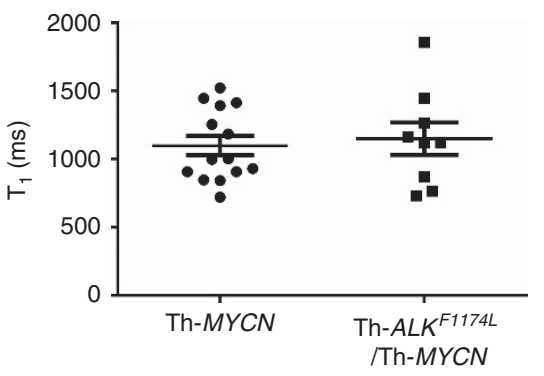

F

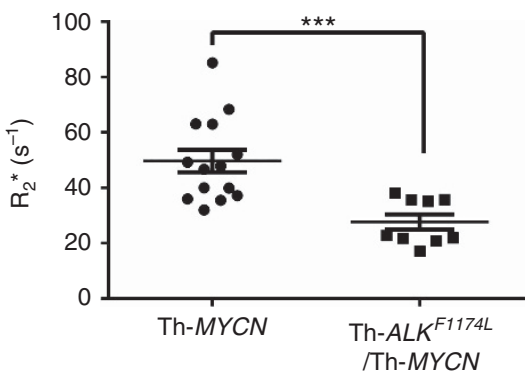

Figure 3. Functional MRI of neuroblastomas at 3 and 7T. (A) Parametric $R_{2}{ }^{*}$ maps acquired at 3 and $7 T$ from the same Th-MYCN transgenic mouse bearing an abdominal neuroblastoma. Note the difference in dynamic range used to display the $R_{2}{ }^{*}$ maps at the two field strengths. (B) Linear regression analysis of $R_{2}{ }^{*}$ measurements obtained from tumour-bearing transgenic mice imaged at both 3 and $7 T$ revealed a significant positive correlation $(R=0.87, P=0.0012)$. Parametric (C) $T_{1}$ and (D) $R_{2}{ }^{*}$ maps acquired at $3 T$ from representative tumour-bearing Th-MYCN and Th-ALK ${ }^{F 1174 L} / T h-M Y C N$ transgenic mice. Summary plots showing the quantitative data for $(E)$ native $T_{1}$ and $(F) R_{2}{ }^{*}$. Data are individual tumour medians, and the mean \pm 1 s.e.m., ${ }^{\star \star \star} P<0.001$.

methods of $T_{1}$ quantification (multiple slice variable flip angle approach at $3 \mathrm{~T} v s$ single slice inversion-recovery at $7 \mathrm{~T}$ ), impossibility of achieving the exact same positioning and acquiring data from the same central slice on the two imaging platforms, and the absence of any $B_{1}$ correction for the data acquired at 3T.

Parametric maps of native $T_{1}$ and $R_{2}{ }^{*}$ obtained at 3T from representative Th-MYCN and Th- $A L K^{F 1174 L} /$ Th-MYCN mice are shown in Figure 3C and D. A more homogeneous distribution of both $T_{1}$ and $R_{2}{ }^{*}$ values was apparent in tumours arising in the Th- $A L K^{F 1174 L} /$ Th-MYCN mice. The quantitative data are summarised in Figure $3 \mathrm{E}$ and $\mathrm{F}$. While there was no significant difference in native tumour $T_{1}$ between the Th-MYCN and the Th$A L K^{F 1174 L} / \mathrm{Th}-M Y C N$ mice $(1098 \pm 71 \mathrm{~ms}$ and $1149 \pm 118 \mathrm{~ms}$; $P>0.1), R_{2}{ }^{*}$ was significantly faster in tumours in the Th-MYCN mice when compared to the Th-ALK $K^{F 1174 L} / \mathrm{Th}-M Y C N$ cohort $\left(49.7 \pm 4 \mathrm{~s}^{-1}\right.$ and $\left.27.7 \pm 3 \mathrm{~s}^{-1} ; P=0.0007\right)$.

DCE-MRI was successfully performed on three transgenic mice simultaneously using the $3 \mathrm{~T}$ platform, yielding data with sufficient temporal and spatial resolution to evaluate vascular biomarkers of each imaged tumour in vivo. Pharmacokinetic modelling was employed to derive and spatially map DCE-MRI parameters. Parametric $K^{\text {trans }}$ maps obtained from each tumour-bearing mouse are shown in Figure 4, along with representative contrast agent uptake curves obtained from ROIs positioned over the tumour, kidney and paraspinal muscle. The mean of median and range values of $K^{\text {trans }}, V_{\mathrm{e}}$ and IAUGC $_{60}$ acquired from the three simultaneously imaged animals were $0.161 \mathrm{~min}^{-1}(0.124-$ $\left.0.202 \mathrm{~min}^{-1}\right), 0.396(0.374-0.415)$ and 3.28 (2.38-4.27) mM.s, respectively.

Anatomical images and parametric maps acquired from representative Th-MYCN mice prior to and $24 \mathrm{~h}$ after treatment with saline or CPM are shown alongside individual tumour volume, $T_{1}$ and $R_{2}{ }^{*}$ values in Figure $5 \mathrm{~A}-\mathrm{C}$, and summarised in Figure 5D. Treatment with CPM elicited a significant $(P=0.0015)$ reduction in tumour burden, and was associated with a significant decrease in native $T_{1}(P=0.0085)$. Collectively this translated to a significant difference in both relative tumour volume $(P=0.0004)$ and native $T_{1}(P=0.0035)$ between the control and CPM groups (Figure 5D). There was no significant treatment-induced change in any parameter over $24 \mathrm{~h}$ in the control cohort. Treatment with CBZ elicited marked tumour growth delay compared to control, resulting in a significant difference in the relative tumour volume $(P=0.0076)$. There was however no associated significant change in relative native $T_{1}$ or $R_{2}{ }^{*}$ with CBZ, and no significant treatmentinduced change in any parameter over $48 \mathrm{~h}$ in the control group (summarised in Figure 5E). 


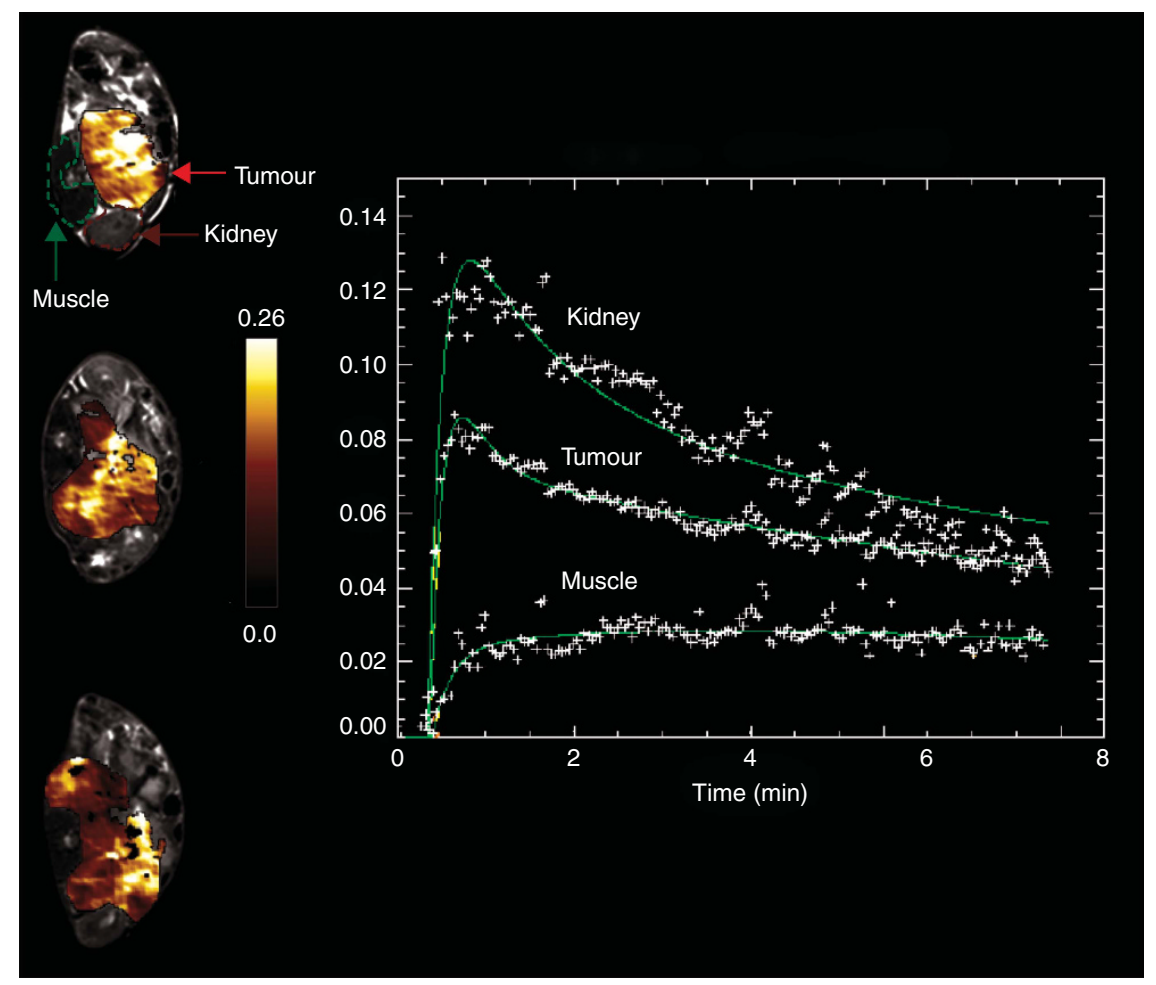

Figure 4. Simultaneous DCE-MRI at 3T. Parametric $K^{\text {trans }}$ maps obtained from three tumour-bearing transgenic mice from which DCE-MRI data were simultaneously acquired using the high resolution 3-channel/3-animal MR RF coil on the 3T clinical scanner, showing a heterogeneous distribution of vascular permeability/perfusion across all three neuroblastomas. Gadolinium uptake curves obtained from one mouse for ROIs positioned over the tumour, kidney and paraspinal muscle are shown. Median $K^{\text {trans }}$ values of $0.202,0.168$ and 0.114 min $^{-1}$ were estimated from the upper, middle and lower tumour ROls, respectively.

\section{DISCUSSION}

Pre-existing and new clinical MRI scanners operating between 1 and $3 \mathrm{~T}$, available to clinically orientated research groups, are being increasingly used for pre-clinical imaging studies in rodents (Dazai et al, 2004; Brockmann et al, 2007; Chen et al, 2007; Inderbitzin et al, 2007; Linn et al, 2007; Nieman et al, 2007; Jerome et al, 2016). The potential and advantages of simultaneous MRI of multiple tumour-bearing animals has been highlighted (Dazai et al, 2004; Li et al, 2005; Beuf et al, 2006; Wilmes et al, 2007). Such pre-clinical applications require the use of appropriately adapted or dedicated RF coils for small fields of view, especially when the acquisition of functional MRI data is considered. For dedicated clinical MRI research centres, the use of such a coil arrangement for performing pre-clinical studies is however more cost-effective compared to low-field benchtop MRI systems that are becoming increasingly available for routine pre-clinical investigations. The necessity to generate linear magnetic field gradients over greater volume on clinical systems limits the geometrical design and hence gradient system performance in comparison to dedicated pre-clinical systems. As a consequence, maximum gradient strengths and slew rates are typically lower, which is also often desirable in order to reduce patient peripheral nerve stimulation. It can however limit EPI-based acquisitions such as DWI, rapid or ultra-high resolution imaging.

In this study, the utility and sensitivity of anatomical and functional MRI data, acquired using a high resolution RF coil on a clinical 3T scanner, from two transgenic mouse models of neuroblastoma was evaluated. In addition, the data were crossreferenced to multiparametric data acquired from the same transgenic mice on a dedicated pre-clinical 7T system, with a particular focus on quantification of native longitudinal relaxation time $T_{1}$, and transverse relaxation rate $R_{2}{ }^{*}$, previously shown to be sensitive imaging biomarkers of treatment response and geno/ phenotype respectively (Jamin et al, 2013, 2014).

Use of the high resolution 3-channel/3-animal RF coil on a clinical $3 \mathrm{~T}$ platform yielded high quality $T_{2}$-weighted anatomical images of up to three mice simultaneously, with sufficient resolution to accurately define and quantify the volume of neuroblastomas arising within the abdomen of the transgenic mice in vivo. The limited region of homogeneous field on dedicated high field pre-clinical systems usually limits the acquisition of functional imaging data from just a few representative slices across the centre of a tumour. This is not the case for clinical systems which allow functional measurements to be made across the whole tumour. In addition, the multiple coil arrangement enabling simultaneous scanning of several subjects enables high throughput ideally suited for efficient cohort screening.

In addition, acquisition of whole mouse body data enables the detection of any distant metastasis in the same imaging session, a clear advantage when compared to the imaging performed on high-field pre-clinical scanners with a more limited region of magnetic field homogeneity. In cancer research, the increasing development and use of more sophisticated orthotopic and transgenic mouse models of primary and secondary disease demands accurate non-invasive imaging methods to confirm successful engraftment/propagation and longitudinal monitoring of deep-seated tumours in vivo (Bielen et al, 2012; Jamin et al, 2013; Graham et al, 2014). The coil arrangement used herein can clearly be used to facilitate such tumour model development, provides a potential screening tool to confirm tumourigenesis, and may alleviate demand at research establishments where the availability/capacity for high-field imaging is limited. 


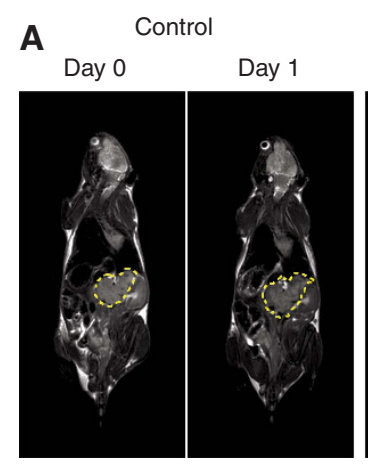

$25 \mathrm{mg} / \mathrm{kg}$ CPM
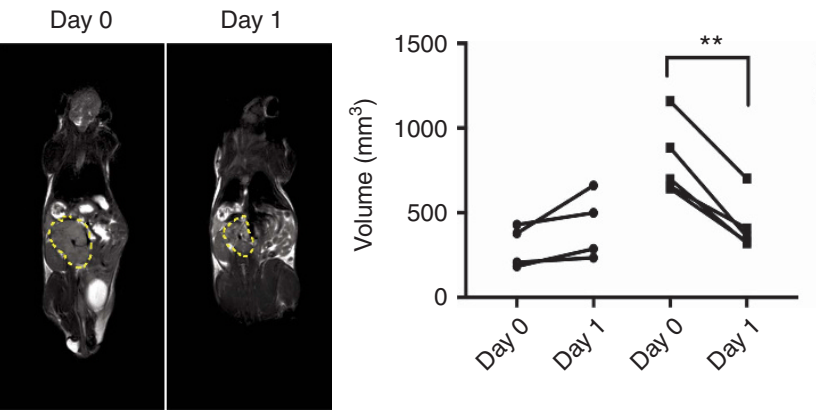
- Control
- $25 \mathrm{mg} / \mathrm{kg}$

CPM

B

Day 0

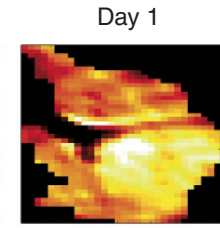

$>1500 \mathrm{~ms}$

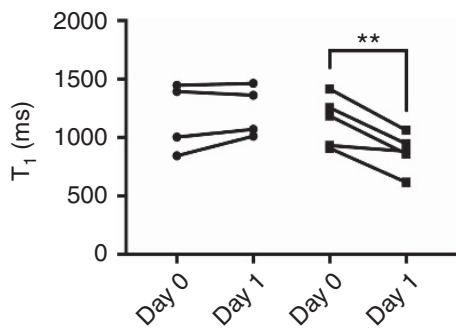
$\rightarrow$ Control
$25 \mathrm{mg} / \mathrm{kg}$
$\mathrm{CPM}$

Contro
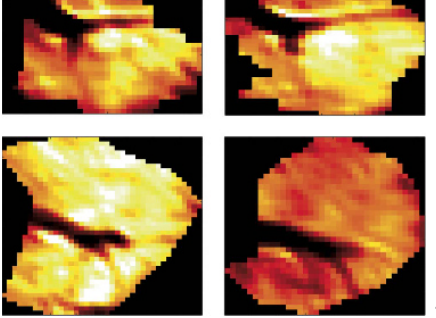

$<500 \mathrm{~ms}$

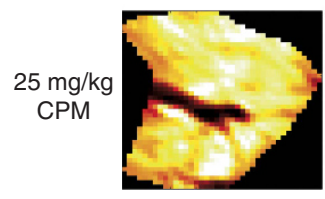

Day 0

C

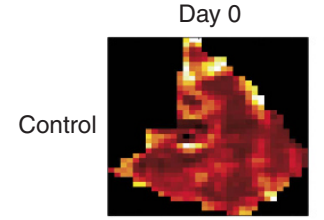

Day 1
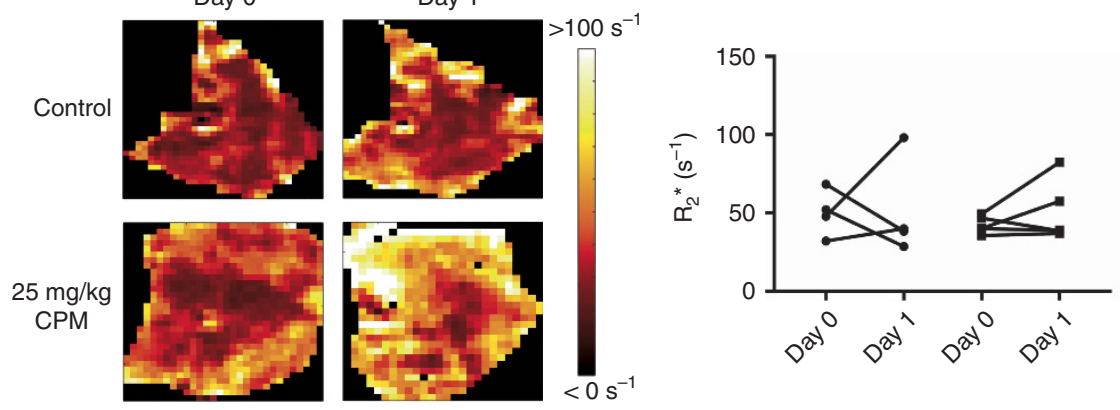

$$
\begin{aligned}
& - \text { Control } \\
& -25 \mathrm{mg} / \mathrm{kg} \\
& \text { CPM }
\end{aligned}
$$
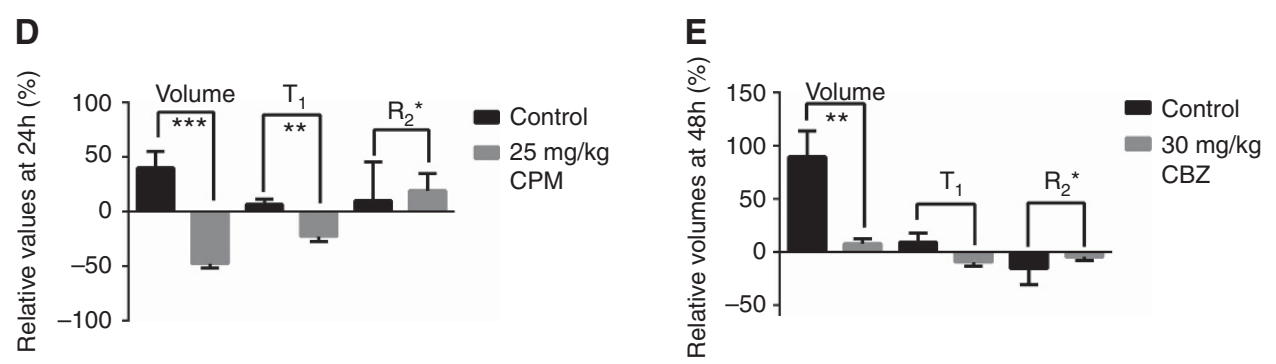

Figure 5. Assessment of tumour response to CPM or CBZ treatment using the $3 T$ platform. (A) $T_{2}$-weighted coronal images acquired at $3 T$ using the high resolution 3-channel/3-animal MR RF coil from Th-MYCN transgenic mice bearing abdominal neuroblastomas prior to and $24 \mathrm{~h}$ after treatment with either saline (control) or CPM. Each tumour is delineated in yellow. Treatment with CPM resulted in a consistent and significant ( $\left.{ }^{\star} P<0.01\right)$ reduction in tumour volume. Parametric maps of tumour $(\mathrm{B})$ native $T_{1}$ and $(\mathrm{C}) \mathrm{R}_{2}{ }^{*}$ acquired at $3 \mathrm{~T}$ from Th-MYCN mice prior to and $24 \mathrm{~h}$ after treatment with either saline (control) or CPM. While treatment with CPM elicited a significant reduction in native $T_{1}$ (** $P<0.01$ ), there was no change in tumour $R_{2}{ }^{*}$. Relative changes in tumour volume, native $T_{1}$ and $R_{2}{ }^{*}$ determined from Th-MYCN mice treated with vehicle, or either CPM

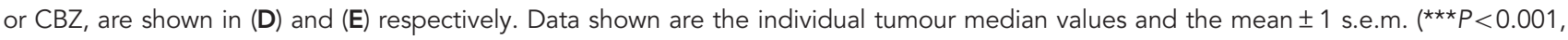
$\star * P<0.01)$.

The ability to quantify functional MRI data using the 3-channel/ 3-animal RF coil at $3 \mathrm{~T}$ was also explored. The primary objective was quantification of tumour native $T_{1}$ and $R_{2}^{*}$, imaging biomarkers previously shown to be sensitive to successful treatment response and haemodynamic vasculature within the Th-MYCN and Th- $A L K^{F 1174 L} /$ Th-MYCN mice, measured at $7 \mathrm{~T}$ (Jamin et al, 2013, 2014). A key aim was to achieve a good compromise between sufficient resolution, SNR and scan time for quantitation of native $T_{1}$ and $R_{2}{ }^{*}$, rather than replicating sequences and parameters routinely used on the $7 \mathrm{~T}$ system. The excellent linearity and positive correlation determined using the $T_{1}$ phantom, and the strong significant correlation of tumour $R_{2}^{*}$ values in vivo, determined across both scanners, suggests that the coil has sufficient sensitivity to acquire and accurately measure native $T_{1}$ and $R_{2}{ }^{*}$ in these transgenic mouse models of neuroblastoma. As expected, the native $T_{1}$ relaxation times in tumours arising within the Th-MYCN mice were lower at $3 \mathrm{~T}$ than those previously reported at 7T (Sasaki et al, 2005; Jamin et al, 
2013). The $R_{2}^{*}$ values acquired in Th-MYCN and Th- $A L K^{F 1174 L} /$ Th-MYCN were $\sim 43 \%$ lower than those determined at $7 \mathrm{~T}$ in this study, are consistent with previously published values (Jamin et al, 2014), and with those reported from human tumours at $3 \mathrm{~T}$ (Hallac et al, 2012; Li et al, 2015; Bane et al, 2016; Panek et al, 2016). A ratio of 2.14 was found between tumour $R_{2}{ }^{*}$ measured at 3 and $7 \mathrm{~T}$ (Figure $3 \mathrm{~B}$ ), which is in agreement with the expected linear increase of the deoxyhaemoglobin $R_{2}{ }^{*}$ relaxivity with magnetic field strength. An $R_{2}{ }^{*}$ ratio of 2.98 has been previously reported for pure blood at 3 and 7T (Blockley et al, 2008). Neuroblastoma is inherently hypervascular, hence quantitation of $R_{2}{ }^{*}$, sensitive to paramagnetic deoxyhaemoglobin, using intrinsic susceptibility MRI is being actively investigated both pre-clinically and clinically as a case-specific imaging biomarker for this paediatric cancer. Encouragingly, the significantly slower $R_{2}{ }^{*}$ values determined at 3T in the tumours arising within the Th- $A L K^{F 1174 L} / \mathrm{Th}-M Y C N$ mice is wholly consistent with their impaired haemodynamic vascular phenotype relative to that in the Th-MYCN mice, previously established using intrinsic susceptibility MRI at 7T (Jamin et al, 2014), supporting the concept of 'MRI genotyping' in vivo (Nieman et al, 2007).

Previous studies have described the acquisition of contrastenhanced MRI data from tumour-bearing rodents (individually or in pairs) on clinical scanners, used to inform on either treatment stratification or treatment response ( $\mathrm{Li}$ et al, 2005; Muruganandham et al, 2006; Wilmes et al, 2007; Egeland et al, 2009; Hompland et al, 2012). In our study we demonstrated the feasibility of performing DCE-MRI simultaneously on three mice using the 3-channel/3-animal RF coil at 3T, enabling the successful pharmacokinetic analysis of Gd-contrast uptake curves in tumours and surrounding organs. The use of volume coils with geometries suitable for small rodents enabled high SNR DCE-MRI measurements with sufficient resolution and coverage necessary for pre-clinical tumour imaging. Furthermore, images are fully compatible with processing tools developed for clinical diagnosis and therapy planning software. This enables evaluation of methods used for multi-parametric and sub-regional tumour analysis, signal modelling (i.e. DCE and DWI models), or methodological MR imaging development. In our study, clinically developed software (MRIW, ADEPT) was used to calculate parametric $T_{1}$ and $R_{2}{ }^{*}$ maps, and to perform pharmacokinetic modelling of the DCE-MRI data.

Finally, the utility of the 3-channel/3-animal RF coil for performing pre-clinical interventional trials at $3 \mathrm{~T}$ was investigated by assessing the anatomical and functional MRI response of tumours arising in Th-MYCN transgenic mice to CPM, the current standard treatment of care for children with high-risk neuroblastoma, or CBZ, currently in clinical trials for the treatment of neuroblastoma. Treatment with CPM resulted in a significant decrease in both tumour volume and native $T_{1}$, consistent with previous results acquired at $7 \mathrm{~T}$ (Jamin et al, 2013). Significant reductions in tumour native $T_{1}$ following successful treatment with a range of therapeutic regimes have been reported both preclinically and clinically, hypothesised to be a consequence of the release of proteins and paramagnetic ions into the extracellular space (O'Connor et al, 2009; McSheehy et al, 2010; Jamin et al, 2013). Treatment with CBZ elicited a cytolentic response only. Collectively these data demonstrate the ability to reveal differential tumour response to treatment using this coil arrangement and multiparametric MRI.

There are several methodological and logistical limitations to performing pre-clinical investigations on clinical MRI systems. A reliable and repeatable shim over a small volume of interest poses a challenge, especially if EPI-based techniques (i.e. diffusion weighted imaging) and spectroscopy are considered. This can contribute to a decrease of $R_{2}{ }^{*}$ linearity measured at both fields. However, differences in the positioning of the imaged volumes, and the relatively high tumour heterogeneity apparent in Th-MYCN tumours, should also be considered. There are practical considerations in positioning lines for the remote, simultaneous administration of contrast agents and delivery of gaseous anaesthetic from the scanner control room, hence the use of injectable anaesthesia in these studies. The utility of this coil arrangement for imaging tumours arising in different anatomical sites within other transgenic and orthotopic mouse models of cancer needs to be established. Finally, significant effort must be made to conform to institutional local rules and minimise any health risk associated with cross-contamination, and to recognise the limited access to clinical scanners during normal hospital operating hours.

In conclusion, we have demonstrated the utility and sensitivity of a high resolution 3-channel/3-animal MR RF coil for performing informative anatomical and functional MRI studies in transgenic mouse models of neuroblastoma on a clinical $3 \mathrm{~T}$ system. In this way, simultaneous data acquisition in multiple tumour-bearing animals can provide high efficiency/throughput for both phenotypic characterisation and trials of novel therapeutics, with additional mechanistic insight provided by multiparametric MRI, improving the accuracy of pre-clinical data and facilitating the introduction of functional MRI biomarkers into aligned imagingembedded clinical trials.

ACKNOWLEDGEMENTS

The authors thank Dr Eva Kousi and Mr David Collins for constructive discussions, the radiographers from the Royal Marsden Hospital MRI unit, and staff from the Biological Services Unit for their help. This study was supported by grants from Cancer Research UK and EPSRC to the Cancer Imaging Centre at ICR, in association with the MRC and Department of Health (England) (C1060/A10334 and C1060/A16464), Cancer Research UK (C46/A10588, C7224/A13407, C1060/A12752), NHS funding to the NIHR Biomedicine Research Centre and the Clinical Research Facility in Imaging, The Wellcome Trust (091763Z/10/Z), and an EPSRC Platform Grant (EP/H046526/1).

\section{CONFLICT OF INTEREST}

The authors declare no conflict of interest.

\section{REFERENCES}

Bane O, Besa C, Wagner M, Oesingmann N, Zhu H, Fiel MI, Taouli B (2016) Feasibility and reproducibility of BOLD and TOLD measurements in the liver with oxygen and carbogen gas challenge in healthy volunteers and patients with hepatocellular carcinoma. J Magn Reson Imaging 43(4): 866-876.

Benjaminsen IC, Graff BA, Brurberg KG, Rofstad EK (2004) Assessment of tumor blood perfusion by high-resolution dynamic contrast-enhanced MRI: a preclinical study of human melanoma xenografts. Magn Reson Med 52(2): 269-276

Berry T, Luther W, Bhatnagar N, Jamin Y, Poon E, Sanda T, Pei D, Sharma B, Vetharoy WR, Hallsworth A, Ahmad Z, Barker K, Moreau L, Webber H, Wang W, Liu Q, Perez-Atayde A, Rodig S, Cheung N-K, Raynaud F, Hallberg B, Robinson SP, Gray NS, Pearson ADJ, Eccles SA, Chesler L, George Rani E (2012) The ALK $K^{F 117 L}$ mutation potentiates the oncogenic activity of MYCN in neuroblastoma. Cancer Cell 22(1): 117-130.

Beuf O, Jaillon F, Saint-Jalmes H (2006) Small-animal MRI: signal-to-noise ratio comparison at 7 and $1.5 \mathrm{~T}$ with multiple-animal acquisition strategies. Magn Reson Mater Phys, Biol Med 19(4): 202-208.

Bielen A, Box G, Perryman L, Bjerke L, Popov S, Jamin Y, Jury A, Valenti M, Brandon AdH, Martins V, Romanet V, Jeay S, Raynaud FI, Hofmann F, Robinson SP, Eccles SA, Jones C (2012) Dependence of Wilms tumor cells 
on signaling through insulin-like growth factor 1 in an orthotopic xenograft model targetable by specific receptor inhibition. Proc Natl Acad Sci USA 109(20): E1267-E1276.

Bilgen M (2013) Feasibility and merits of performing preclinical imaging on clinical radiology and nuclear medicine systems. Int J Mol Imaging 2013: 923823.

Blockley NP, Jiang L, Gardener AG, Ludman CN, Francis ST, Gowland PA (2008) Field strength dependence of R1 and $\mathrm{R} 2^{*}$ relaxivities of human whole blood to prohance, vasovist, and deoxyhemoglobin. Magn Reson Med 60(6): 1313-1320.

Brockmann MA, Kemmling A, Groden C (2007) Current issues and perspectives in small rodent magnetic resonance imaging using clinical MRI scanners. Methods 43(1): 79-87.

Chen F, De Keyzer F, Wang H, Vandecaveye V, Landuyt W, Bosmans H, Hermans R, Marchal G, Ni Y (2007) Diffusion weighted imaging in small rodents using clinical MRI scanners. Methods 43(1): 12-20.

Chesler L, Weiss WA (2011) Genetically-engineered murine models contribution to our understanding of the genetics, molecular pathology and therapeutic targeting of neuroblastoma. Semin Cancer Biol 21(4): 245-255.

d'Arcy JA, Collins DJ, Padhani AR, Walker-Samuel S, Suckling J, Leach MO (2006) Magnetic resonance imaging workbench: analysis and visualization of dynamic contrast-enhanced MR imaging data. RadioGraphics 26(2): 621-632.

Dazai J, Bock NA, Nieman BJ, Davidson LM, Henkelman RM, Chen XJ (2004) Multiple mouse biological loading and monitoring system for MRI. Magn Reson Med 52(4): 709-715.

de Graaf RA, Brown PB, McIntyre S, Nixon TW, Behar KL, Rothman DL (2006) High magnetic field water and metabolite proton T1 and T2 relaxation in rat brain in vivo. Magn Reson Med 56(2): 386-394.

de Jong M, Essers J, van Weerden WM (2014) Imaging preclinical tumour models: improving translational power. Nat Rev Cancer 14(7): 481-493.

Doran SJ, d'Arcy J, Collins DJ, Andriantsimiavona R, Orton M, Koh D-M, Leach MO (2012) Informatics in radiology: development of a research PACS for analysis of functional imaging data in clinical research and clinical trials. RadioGraphics 32(7): 2135-2150.

Egeland TAM, Simonsen TG, Gaustad J-V, Gulliksrud K, Ellingsen C, Rofstad EK (2009) Dynamic contrast-enhanced magnetic resonance imaging of tumors: preclinical validation of parametric images. Radiat Res 172(3): 339-347.

Fram EK, Herfkens RJ, Johnson GA, Glover GH, Karis JP, Shimakawa A, Perkins TG, Pelc NJ (1987) Rapid calculation of T1 using variable flip angle gradient refocused imaging. Magn Reson Imaging 5(3): 201-208.

Graham TJ, Box G, Tunariu N, Crespo M, Spinks TJ, Miranda S, Attard G, de Bono J, Eccles SA, Davies FE, Robinson SP (2014) Preclinical evaluation of imaging biomarkers for prostate cancer bone metastasis and response to cabozantinib. J Natl Cancer Inst 106(4): dju033.

Hallac RR, Ding Y, Yuan Q, McColl RW, Lea J, Sims RD, Weatherall PT, Mason RP (2012) Oxygenation in cervical cancer and normal uterine cervix assessed using blood oxygenation level-dependent (BOLD) MRI at 3T. NMR Biomed 25(12): 1321-1330.

Hompland T, Ellingsen C, Rofstad E (2012) Preclinical evaluation of Gd-DTPA and gadomelitol as contrast agents in DCE-MRI of cervical carcinoma interstitial fluid pressure. BMC Cancer 12(1): 544 .

Inderbitzin D, Stoupis C, Sidler D, Gass M, Candinas D (2007) Abdominal magnetic resonance imaging in small rodents using a clinical $1.5 \mathrm{~T}$ MR scanner. Methods 43(1): 46-53.

Jamin Y, Glass L, Hallsworth A, George R, Koh D-M, Pearson ADJ, Chesler L, Robinson SP (2014) Intrinsic susceptibility MRI identifies tumors with $A L K^{F 1174 L}$ mutation in genetically-engineered murine models of high-risk neuroblastoma. PLoS ONE 9(3): e92886.

Jamin Y, Tucker ER, Poon E, Popov S, Vaughan L, Boult JKR, Webber H, Hallsworth A, Baker LCJ, Jones C, Koh D-M, Pearson ADJ, Chesler L, Robinson SP (2013) Evaluation of clinically translatable MR imaging biomarkers of therapeutic response in the TH-MYCN transgenic mouse model of neuroblastoma. Radiology 266(1): 130-140.

Jerome NP, Boult JKR, Orton MR, d'Arcy J, Collins DJ, Leach MO, Koh D-M, Robinson SP (2016) Modulation of renal oxygenation and perfusion in rat kidney monitored by quantitative diffusion and blood oxygen level dependent magnetic resonance imaging on a clinical $1.5 \mathrm{~T}$ platform. $B M C$ Nephrol 17(1): 142.
Kilkenny C, Browne WJ, Cuthill IC, Emerson M, Altman DG (2010) Improving bioscience research reporting: the ARRIVE guidelines for reporting animal research. PLoS Biol 8(6): e1000412.

Li K-L, Wilmes LJ, Henry RG, Pallavicini MG, Park JW, Hu-Lowe DD, McShane TM, Shalinsky DR, Fu YJ, Brasch RC, Hylton NM (2005) Heterogeneity in the angiogenic response of a BT474 human breast cancer to a novel vascular endothelial growth factor-receptor tyrosine kinase inhibitor: Assessment by voxel analysis of dynamic contrast-enhanced MRI. J Magn Reson Imaging 22(4): 511-519.

Li XS, Fan HX, Fang H, Song YL, Zhou CW (2015) Value of R2* obtained from $\mathrm{T} 2 *$-weighted imaging in predicting the prognosis of advanced cervical squamous carcinoma treated with concurrent chemoradiotherapy. J Magn Reson Imaging 42(3): 681-688.

Linn J, Schwarz F, Schichor C, Wiesmann M (2007) Cranial MRI of small rodents using a clinical MR scanner. Methods 43(1): 2-11.

Maris JM (2010) Recent advances in neuroblastoma. N Engl J Med 362(23): 2202-2211.

McSheehy PMJ, Weidensteiner C, Cannet C, Ferretti S, Laurent D, Ruetz S, Stumm M, Allegrini PR (2010) Quantified tumor $\mathrm{T}_{1}$ is a generic early-response imaging biomarker for chemotherapy reflecting cell viability. Clin Cancer Res 16(1): 212-225.

Muruganandham M, Lupu M, Dyke JP, Matei C, Linn M, Packman K, Kolinsky K, Higgins B, Koutcher JA (2006) Preclinical evaluation of tumor microvascular response to a novel antiangiogenic/antitumor agent RO0281501 by dynamic contrast-enhanced MRI at 1.5T. Mol Cancer Therapeut 5(8): 1950-1957.

Nieman BJ, Bishop J, Dazai J, Bock NA, Lerch JP, Feintuch A, Chen XJ, Sled JG, Henkelman RM (2007) MR technology for biological studies in mice. NMR Biomed 20(3): 291-303.

O'Connor JPB, Carano RAD, Clamp AR, Ross J, Ho CCK, Jackson A, Parker GJM, Rose CJ, Peale FV, Friesenhahn M, Mitchell CL, Watson Y, Roberts C, Hope L, Cheung S, Reslan HB, Go MAT, Pacheco GJ, Wu X, Cao TC, Ross S, Buonaccorsi GA, Davies K, Hasan J, Thornton P, del Puerto O, Ferrara N, van Bruggen N, Jayson GC (2009) Quantifying antivascular effects of monoclonal antibodies to vascular endothelial growth factor: insights from imaging. Clin Cancer Res 15(21): 6674-6682.

Panek R, Welsh L, Dunlop A, Wong KH, Riddell AM, Koh D-M, Schmidt MA, Doran S, McQuaid D, Hopkinson G, Richardson C, Nutting CM, Bhide SA, Harrington KJ, Robinson SP, Newbold KL, Leach MO (2016) Repeatability and sensitivity of $\mathrm{T}^{*}$ measurements in patients with head and neck squamous cell carcinoma at 3T. J Magn Reson Imaging 44(1): 72-80.

Rasmuson A, Segerström L, Nethander M, Finnman J, Elfman LHM, Javanmardi N, Nilsson S, Johnsen JI, Martinsson T, Kogner P (2012) Tumor development, growth characteristics and spectrum of genetic aberrations in the TH-MYCN mouse model of neuroblastoma. PLOS One 7(12): e51297.

Sasaki M, Shibata E, Kanbara Y, Ehara S (2005) Enhancement effects and relaxivities of gadolinium-DTPA at 1.5 versus 3 Tesla: a phantom study. Magn Reson Med Sci 4(3): 145-149.

Tofts PS, Brix G, Buckley DL, Evelhoch JL, Henderson E, Knopp MV, Larsson HBW, Lee T-Y, Mayr NA, Parker GJM, Port RE, Taylor J, Weisskoff RM (1999) Estimating kinetic parameters from dynamic contrast-enhanced T1-weighted MRI of a diffusable tracer: Standardized quantities and symbols. J Magn Reson Imaging 10(3): 223-232.

Ullman P, Junge S, Cowin G, Luescher K, Hennig J (2004) High field parallel imaging in small rodents. In Proceedings of the ISMRM, p 1610.

Walker-Samuel S, Orton M, McPhail LD, Boult JKR, Box G, Eccles SA, Robinson SP (2010) Bayesian estimation of changes in transverse relaxation rates. Magn Reson Med 64(3): 914-921.

Wech T, Lygate CA, Neubauer S, Köstler H, Schneider JE (2012) Highly accelerated cardiac functional MRI in rodent hearts using compressed sensing and parallel imaging at 9.4T. J Cardiovasc Magn Reson 14(1): P65.

Weiss WA, Aldape KD, Mohapatra G, Feuerstein BG, Bishop JM (1997) Targeted expression of MYCN causes neuroblastoma in transgenic mice. EMBO J 1611: 2985-2995.

Wilmes LJ, Pallavicini MG, Fleming LM, Gibbs J, Wang D, Li K-L, Partridge SC, Henry RG, Shalinsky DR, Hu-Lowe D, Park JW, McShane TM, Lu Y, Brasch RC, Hylton NM (2007) AG-013736, a novel inhibitor of VEGF receptor tyrosine kinases, inhibits breast cancer growth and decreases vascular permeability as detected by dynamic contrast- 
enhanced magnetic resonance imaging. Magn Reson Imaging 25(3): 319-327.

Workman P, Aboagye EO, Balkwill F, Balmain A, Bruder G, Chaplin DJ, Double JA, Everitt J, Farningham DAH, Glennie MJ, Kelland LR,

Robinson V, Stratford IJ, Tozer GM, Watson S, Wedge SR, Eccles SA (2010) Guidelines for the welfare and use of animals in cancer research. Br J Cancer 102(11): 1555-1577.

Yakes FM, Chen J, Tan J, Yamaguchi K, Shi Y, Yu P, Qian F, Chu F, Bentzien F, Cancilla B, Orf J, You A, Laird AD, Engst S, Lee L, Lesch J, Chou Y-C, Joly AH (2011) Cabozantinib (XL184), a novel MET and
VEGFR2 inhibitor, simultaneously suppresses metastasis, angiogenesis, and tumor growth. Mol Cancer Therapeut 10(12): 2298-2308.

(c) (i) This work is licensed under the Creative Commons Attribution 4.0 International License. To view a copy of this license, visit http://creativecommons.org/licenses/by/4.0/

C) The Author(s) named above 2017 